\title{
Atomic scale modelling of the cores of dislocations in complex materials part 2: applications $\dagger \ddagger$
}

\author{
Andrew M. Walker, $\S^{* a b}$ Julian D. Gale, $^{c}$ Ben Slater ${ }^{a}$ and Kate Wright ${ }^{a b c d}$ \\ ${ }^{a}$ Davy Faraday Research Laboratory, The Royal Institution of Great Britain, 21 Albemarle \\ Street, London, UKW1S 4BS.E-mail: andrew.m.walker@anu.edu.au; \\ Fax: +61 (0)2 6125 8253; Tel: +61 (0)2 61254076 \\ ${ }^{b}$ Department of Earth Sciences, University College London, Gower Street, London, \\ UK WC1E $6 B T$ \\ ${ }^{c}$ Nanochemistry Research Institute, Department of Applied Chemistry, Curtin University of \\ Technology, P.O. Box U1987, Perth, 6845, Western Australia \\ ${ }^{d}$ Department of Chemistry, Christopher Ingold Laboratories, University College London, \\ 20 Gordon Street, London, UK WCIH OAJ
}

Received 22nd April 2005, Accepted 6th July 2005

First published as an Advance Article on the web 3rd August 2005

In an accompanying article, we have described a methodology for the simulation of dislocations in structurally complex materials. We illustrate the applicability of this method through studies of screw dislocations in a structurally simple ionic ceramic $(\mathrm{MgO})$, a molecular ionic mineral (forsterite, $\mathrm{Mg}_{2} \mathrm{SiO}_{4}$ ), a semi-ionic zeolite (siliceous zeolite A) and a covalent molecular crystalline material (the pharmaceutical, orthorhombic paracetamol-II). We focus on the extent of relaxation and the structure of the dislocation cores and comment on similarities and points of disparity between these materials. It is found that the magnitude of the relaxation varies from material to material and does not simply correlate with the magnitude of the principal elastic constants in an easily predictable fashion, or with the size of the cohesive lattice energy or length of the Burgers vector, which emphasises the need to model the non-linear forces and atomic structure of the core.

\section{Introduction}

In the preceding paper, we described a method for the simulation of the cores of dislocations in ionic and semi-ionic crystals, with many ions in their basis. The dislocation core, and its immediate surroundings are modelled using a parameterised model of the interatomic interactions. This model is embedded in an elastic description of the infinite crystal represented by atoms that are held fixed during the simulation. The model is periodic along the dislocation line and all simulations are performed using the latest revision of the GULP code. ${ }^{2}$

Here we describe studies of screw dislocations in a structurally diverse range of materials, namely $\mathrm{MgO}$, forsterite $\left(\mathrm{Mg}_{2} \mathrm{SiO}_{4}\right.$; a silicate mineral found in large concentrations in the Earth's upper mantle), siliceous zeolite A and paracetamol (a major pain reducing drug). We focus on the structure of the dislocation cores and find qualitative differences between dislocations in dense and open-framework materials.

\section{Screw dislocations in $\mathrm{MgO}$}

$\mathrm{MgO}$ has been the subject of a vast number of computational studies and is the classic test system for models of ionic materials, as well as being an important mineral phase in the Earth's deep interior. Although it has a simple structure, some of its properties are not easy to model. In particular, the elastic constants violate Cauchy's relation, which states that, for

\section{$\dagger$ For part 1 see ref. 1}

† Electronic supplementary information (ESI) available: Figs. S1-S6 (see text) and xyz files of the structures of the dislocations in Figs. 6, 7 and 10. See http://dx.doi.org/10.1039/b505716g

$\S$ Current address: Research School of Earth Sciences, Australian National University, Mills Road, Canberra, ACT 0200, Australia. simple cubic materials with central pair-wise forces acting between ions, $\mathrm{C}_{12}$ must be equal to $\mathrm{C}_{44}$. This leads to the conclusion that there are significant many body interactions in $\mathrm{MgO}$, or that there are off-centred forces acting between pairs of ions. Parameterised potential models must consider this effect if they are to reproduce the elastic constant tensor of $\mathrm{MgO}$. There are a wide range of potential models for $\mathrm{MgO}$, including those which make use of the shell model to describe the oxygen ions ${ }^{3}$ and those that make use of a breathing shell model. ${ }^{4}$ Use of the shell model alone results in good reproduction of the dielectric behaviour, but fails to correctly describe the elastic properties. However, breathing shell models can correctly reproduce the Cauchy violation and give the correct elastic behaviour. For this reason, the breathing shell model of Gale and Rohl ${ }^{2}$ was used for studies of dislocations in $\mathrm{MgO}$, which gives a good description of the structure, elastic and dielectric behaviour of $\mathrm{MgO}$.

The structure of $\mathrm{MgO}$ leads to several important slip systems, with easy slip observed for the $\langle 1 \overline{1} 0\rangle\{110\}$ system. Slip of the $\langle 0 \overline{1} 1\rangle\{110\}$ system is also observed when single crystals are loaded along the $\langle 111\rangle$ axis to suppress easy slip, and in polycrystalline samples at high temperature. ${ }^{5}$ The $\langle 100\rangle$ screw dislocation, while apparently not important for the deformation mechanism, is thought to be important for crystal growth. ${ }^{6}$

The $\langle 100\rangle$ screw dislocation, and its effect on the growth of $\mathrm{MgO}$ has been considered by Watson and co-workers. ${ }^{6-8}$ This work utilised the METADISE code and a rigid ion model of $\mathrm{MgO}$. The description of the elastic and dielectric behaviour is therefore expected to be less accurate than in these calculations, but the previous work is invaluable as a benchmark for our method. The methodology Watson and co-workers adopted for the simulation of an isolated screw dislocation is 
subtly different from the scheme outlined in the companion paper. The major difference is in the way the dislocation energy was calculated. Watson et al. ${ }^{7}$ performed a series of geometry optimisations with varying sizes of the free atomistic region surrounded by a fixed size shell of atoms held at the location predicted by linear elasticity. This is in contrast to our approach, and the approach of previous workers such as Puls and Norgett ${ }^{9}$ and Hoagland et al. ${ }^{10}$ where geometry optimisation is performed and then this relaxed geometry is used to calculate the energy. The result of this difference is that any core effects are tightly constrained within the smaller radius cells used by Watson et al. ${ }^{7}$ such that if, for example, the core expands with respect to the bulk material then at small radii there will be a high effective pressure on the core. This effect will decrease with increasing relaxed radius. In practice, this means that the extracted dislocation energy will be calculated at some effective pressure and that the elastic constants cannot be used to derive the elastic part of the dislocation energy. The energy factor extracted by Watson et al. ${ }^{7}$ is a fitted parameter from the data, rather than a fixed value derived from the elastic constants. However, as long as a large cell is selected for the description of the structure, the geometry of the core extracted by Watson et al. ${ }^{7}$ is not expected to differ from our calculations described below. A third difference is that the simulation cells of Watson $\mathrm{et} \mathrm{al.}^{7}$ are of square cross section perpendicular to the dislocation line and our results use a cell with a circular cross section. For two cells of equal radius, the approach described here will require fewer ions within the simulation cell and forces are expected to attenuate in a truly radial fashion. It is worth noting that for $\mathrm{MgO}$, no significant differences in energy or structure between the two methods is expected (for sufficiently large radii), but for other materials, it is possible discrepancies may arise.

For our study, convergence testing was carried out in order to select sizes for the relaxed and fixed regions in the calculation. Fig. 1 shows the effect of increasing the thickness of the fixed region while keeping the free region at a fixed radius. It is clear that convergence is achieved relatively quickly, and a shell of ions $15 \AA$ thick is easily large enough to converge the electrostatic energy of the free region and to isolate it from the surrounding vacuum. Convergence of the free region is not so straightforward because of the logarithmic dependence of energy on system size. A convenient test is to increase the radius of the free region until the outer most atoms do not move during the energy minimisation, the difficulty is that such testing rapidly becomes inefficient. After some initial testing with smaller cells, it was found that a free region of $50 \AA$ radius was thick enough to isolate the non-linear effects of the core from the fixed elastic surrounding region (see Fig. S1 in the ESI, $\ddagger$ for example). Hence, the radius of region 1 was $50 \AA$ and

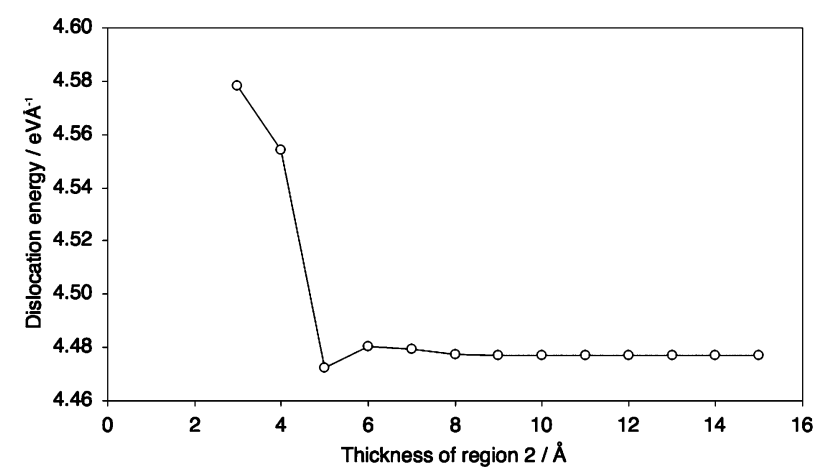

Fig. 1 Convergence of the dislocation energy with thickness of the fixed portion of the simulation cell. The radius of the inner region is $25 \AA$ for the purposes of these convergence tests and the reported dislocation energy is the difference in energy between a dislocated and bulk-like cell stored within the inner region. Ionic relaxation is not taken into account.

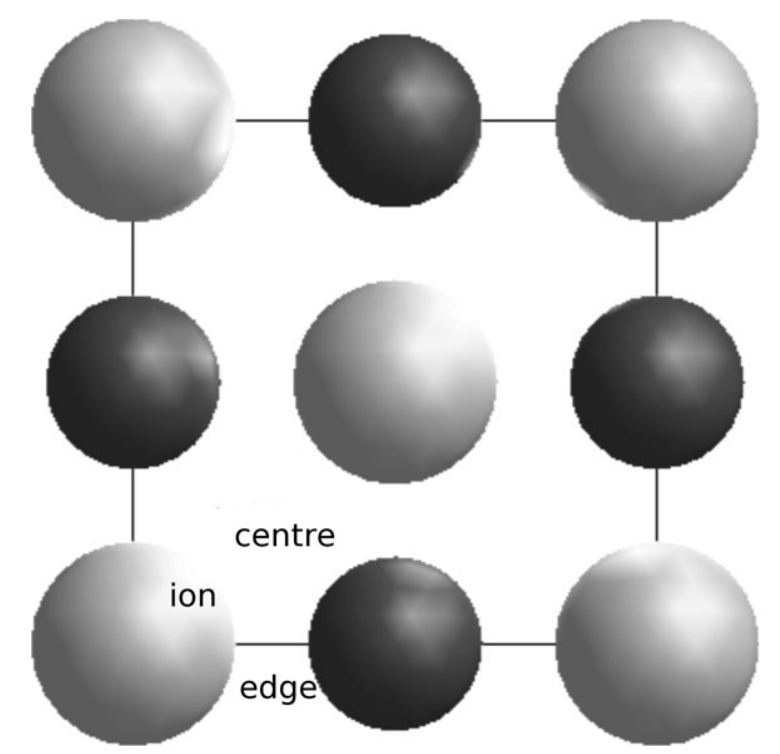

Fig. 2 Three locations used to centre the 1D simulation cell and the elastic displacement field for models of the $\langle 100\rangle$ screw dislocations in $\mathrm{MgO}$. All lie on rotation axes and so minimise the dipole on the simulation cell.

the radius of region 2 (from the centre of the displacement field) was $65 \AA$. There are three obvious possibilities for the location of the origin of the displacement field termed the edge, ion and centre sites shown in Fig. 2. ${ }^{7}$ The cells centred on these sites contained 8994, 8943 and 9000 species (i.e. the sum of the magnesium cores, the oxygen cores and oxygen shells in each cell), respectively. All the cells had a periodic repeat of $4.212 \AA$ (the optimised cell parameter) along the dislocation line. Introduction of the dislocation is relatively straightforward in this case as the anisotropic displacement field reduces to the isotropic form. Energy minimisation was performed using the conjugate gradients method and the Saunders et al. ${ }^{11}$ Coulomb summation was used.

The final energies of the simulation cells are $-13.33 \mathrm{eV}^{-1}{ }^{-1}$ in each of the three cases (compared to a bulk cell value of $-13.35 \mathrm{eV}$ ion $\left.^{-1}\right)$. Clearly the total strain caused by the dislocation and averaged over the whole cell is small, but to extend this to macroscopic crystals it must be remembered that the whole crystal is strained not just the region close the core. The dislocation energy, $E(r)$, (difference between the energy of the bulk and dislocated systems) as a function of radius, $r$, is presented in Fig. 3. This energy is fitted to the elastic solution to the dislocation energy. Fig. 3a shows the critical importance of accounting for anisotropy in this procedure. Two of the three lines represent fits using the Voigt and Reuss definitions of the shear modulus (133.8 and $128.0 \mathrm{GPa}$ respectively) with an isotropic definition of the elastic part of the energy. The gradient of the line on the log plot clearly shows that the isotropic elastic solution does not fit the results of the atomistic solution. Allowing the shear modulus to become an additional fitted parameter, the procedure used by Watson et al., ${ }^{7}$ results in a much better fit, as does fixing the "shear modulus" as the value of the energy factor as derived by Steeds. ${ }^{12}$ Thus the equation used to fit is:

$$
E(r)=E(\text { core })+\frac{\left(S_{44} S_{55}\right)^{-1 / 2} b^{2}}{4 \pi} \ln \left(\frac{r}{r_{0}}\right),
$$

where $E$ (core) is the energy stored in the dislocation core which has a radius $r_{0}, b$ is the length of the Burgers vector and $S_{44}$ and $S_{55}$ are components of the reduced elastic compliance tensor (see the Appendix of the accompanying paper). Taking the anisotropic energy factor results in very good fits with just one independent parameter (obviously the core energy depends on 

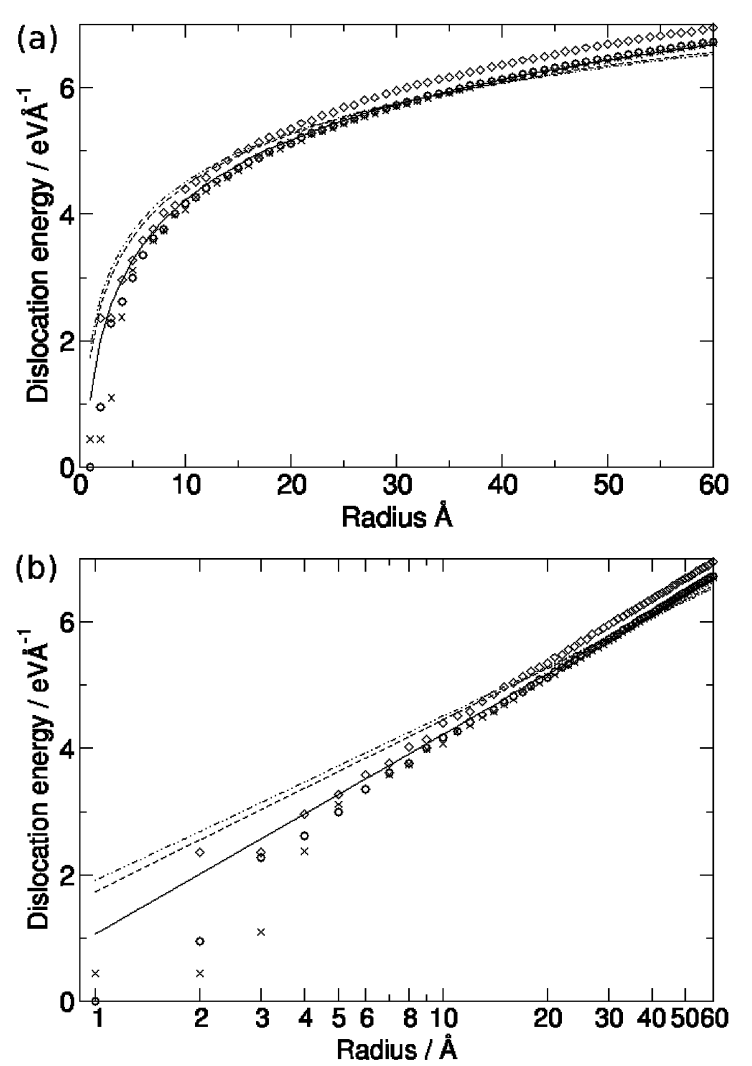

Fig. 3 Energy of $\mathrm{MgO}$ screw dislocation as a function of radius from the dislocation line plotted on a linear (a) and logarithmic (b) scale. Diamonds, circles and crosses represent data points taken from the atomistic model for the centre, ion and edge choices of origin respectively. Solid, dashed and dash and dot lines are fits to the data for the edge site using the anisotropic, Voigt and Reuss definitions respectively (see text for details).

the core radius). Fixing the core radius at $10 \AA$ and only fitting to the data points from the radii in the larger region 1 yields core energies of $4.2213,4.4408$ and $4.1916 \mathrm{eV}^{-1}$ for the edge, centre and ion sites respectively. This is the same ordering of energies found by Watson et al..$^{7}$ despite the different approach.

Fig. 4 shows the final structure of the core of the [100] screw dislocation (all three cells give essentially the same geometry). In analysing this structure, it is useful to compare the final structure with the starting structure as derived by linear elastic theory; Fig. S1 of the electronic supplementary information (ESI) $\ddagger$ shows this difference, which is termed the core displacement field. The core relaxation can conveniently be separated into two parts with distinct causes. The first effect is that the atoms tend to move in towards the core (Fig. S1a). This mode of displacement is because a group of atoms, which originally formed a circle around the incipient dislocation line, form a helix after the introduction of the dislocation. As the elastic displacement field does not contain components perpendicular

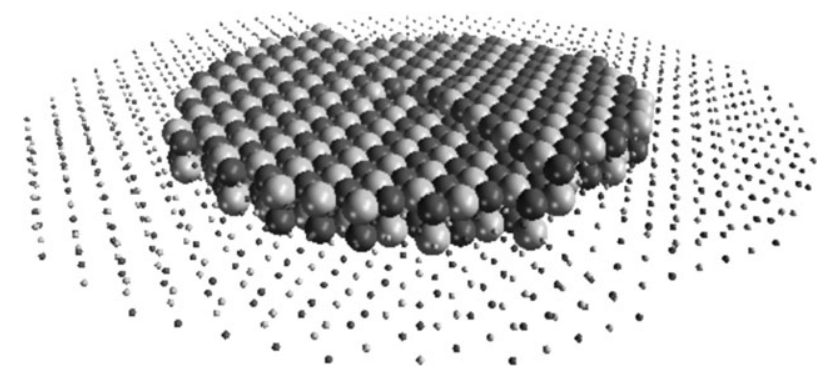

Fig. 4 Relaxed simulation cell containing $\langle 100\rangle$ screw dislocation in $\mathrm{MgO}$ ( $\mathrm{Mg}$ dark grey, $\mathrm{O}$ light grey). The smaller spheres represent ions held fixed in the elastic region and the larger spheres represent atoms that are allowed to relax during the energy minimisation.

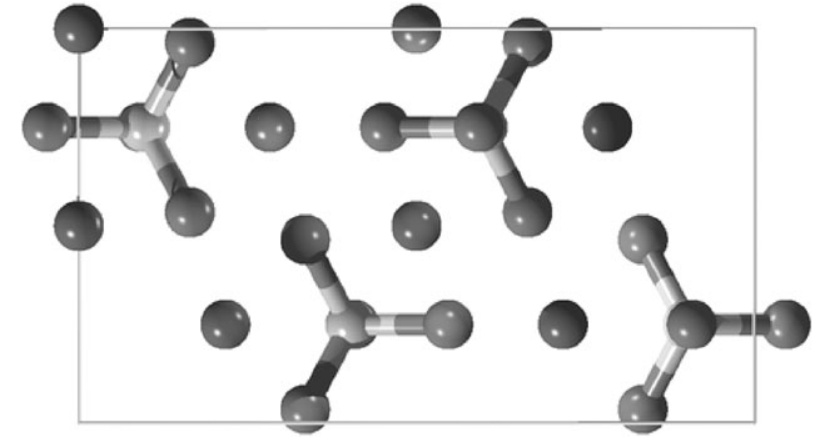

Fig. 5 The olivine unit cell looking along [100] with oxygen in mid grey, magnesium in dark grey and silicon in light grey, shown bonded to the oxygen.

to the dislocation line, the radius of the helix and the original circle are the same, such that all the $\mathrm{Mg}-\mathrm{O}$ bond distances are increased by the process of introducing the dislocation. On relaxation, these bond distances tend to reduce towards their natural values in the bulk, which is achieved by the inwards relaxation. The second mode of displacement is motion parallel to the dislocation line (Fig. S1b). This occurs because of the discontinuity at the origin of the elastic displacement field. For example, an atom a short distance along the positive $x$-axis has a value of $\theta$ of 0 , a short distance along the negative $y$-axis gives a value of $180^{\circ}$, the displacement along $z$ for these two points are 0 and 0.5 times the Burgers vector respectively, crossing the origin leads to a step of $0.5 b$ in the displacement field. In $\mathrm{MgO}$ this discontinuity is equal to the $\mathrm{Mg}-\mathrm{O}$ separation so that like atoms are brought into contact across the discontinuity. This is clearly energetically unfavourable so the atoms close to the core move along $z$ to remove the discontinuity. It is notable that the total displacement in $z$ is equal to $2.4 \AA$, or half the Burgers vector. The resultant twisted structure is reminiscent of the structure of one-dimensional ionic crystals grown inside carbon nanotubes which adopt an unusual packing pattern to avoid atoms of like charge being in close proximity to each other. $^{13}$

\section{Screw dislocations in forsterite}

The magnesium-rich olivine, forsterite $\left(\mathrm{Mg}_{2} \mathrm{SiO}_{4}\right)$, is an important mineral forming $\sim 60 \%$ of the Earth's upper mantle. Structurally, olivine can be described as either an orthosilicate or a hexagonally close packed (HCP) lattice of oxygen ions with half of the octahedral sites occupied by iron or magnesium ions, and one eighth of the tetrahedral sites occupied by silicon ions. These ions distort the HCP lattice and give olivine orthorhombic symmetry (space group Pbnm, Fig. 5). There are three symmetry inequivalent oxygen sites (O1, $\mathrm{O} 2$ and $\mathrm{O} 3)$, two metal sites (M1 and M2, both occupied by magnesium ions in forsterite) and one silicon site. The M1 site is on an inversion centre, while M2 lies on a mirror plane. As the dominant mineral, the deformation of olivine is likely to play a crucial role in determining the rate of deformation of the Earth's mantle, thus controlling the process of plate tectonics. Briefly, there are two proposed strain rate controlling processes which may be operating in mantle olivine; dislocation climb controlled by the diffusion of point defects, ${ }^{14}$ and the cross slip of screw dislocations. ${ }^{15}$ Most workers prefer the former mechanism, but there is no clinching argument for, or against, either of them. The observed slip systems are rather complex, and vary with the strain rate and temperature, but both $[100](0 \mathrm{kl})$ and $[001](h k 0)$ have been widely reported. ${ }^{16}$ So far, the structure of the cores of dislocations in olivine are unknown, which makes the assignment of a deformation mechanism to the Earth's upper mantle difficult because of the great importance of the dislocation core to such processes. 
Here we report the first computational study of the cores of dislocations in any mantle silicate. In particular, models of the structure of the cores of some dislocations in olivine are described. In order to simplify matters, only a subset of the observed dislocations have been modelled, namely the $a[100]$ and $c[001]$ screw dislocations. We use a well tested potential model fitted to the structure of quartz and oxide materials. ${ }^{2,17}$ This model has been extensively used for studies of the thermodynamics, lattice dynamics, point defect properties and surface structure of forsterite. ${ }^{18}$ In order to utilise this potential model the Coulomb summation of Saunders et al. ${ }^{11}$ is used.

$a[100]$ screw dislocations in forsterite have been modelled using the general methodology described in the accompanying paper. The simulation cell chosen was $75 \AA$ in radius $(12025$ atoms) with the outer $25 \AA$ held fixed during the atomistic relaxation. In order to produce a charge neutral simulation cell, we generalised the technique used by Braithwaite et al. ${ }^{19}$ for constructing charge neutral, non-periodic forsterite clusters used for the simulation of point defects. This method makes use of the fact that forsterite consists of octahedrally coordinated magnesium ions and tetrahedrally co-ordinated silicon ions, and that each oxygen ion takes part in the coordination of one silicon ion and three magnesium ions. We therefore consider that each oxygen ion "uses" -1 unit of charge to contribute to neutralising the silicon tetrahedron and $-1 / 3$ of a unit of charge to neutralise each magnesium octahedron. Where the polyhedra touch at the vertices the charge on the oxygen ion consists of the sum the charges used for each polyhedra. In a perfect crystal, each oxygen ion then has a total charge of -2 ( 3 times $-1 / 3$ plus -1$)$. The simulation cell is only constructed out of these neutral $\left[\mathrm{Mg}^{+2} \mathrm{O}_{6}^{-1 / 3}\right]$ and $\left[\mathrm{Si}^{+4} \mathrm{O}_{4}^{-1}\right]$ units, which implies that the edge of the simulation cell is terminated with oxygen ions with partial charges as the oxygen does not contribute to neutralising all four polyhedra as in the bulk. Because each building unit is charge neutral, the whole cell also has no net charge. This method can be used for many other crystal structures but in some materials (for example wadsleyite, a high-pressure polymorph of forsterite containing $\mathrm{Si}_{2} \mathrm{O}_{7}$ units) simple chargeneutral units cannot be unambiguously defined. The cell is centred on the M1 sites to avoid any total dipole. Several sites were selected to act as the origin of the dislocation within this cell and single point calculations indicated that an origin within the M1 "channel" yielded the lowest energy. However, as this site also removes the dipole across the cell (as described below) it is possible that this effect is not directly related to the structure of the core.

The way that the dislocation modifies the electrostatic dipole across the simulation cell is rather interesting. If one considers a thin slice through the cell and calculates the components of its dipole perpendicular to [100], a finite dipole is found. This dipole is exactly cancelled by a dipole in the opposite direction in the equivalent slice displaced by half the lattice parameter along $a$. This arises because the M1 site is an inversion centre. When the screw dislocation is introduced, this offsets the two sides of the cell by $a / 2$ and removes the dipole in any, and every, slice because the string of inversion centres is replaced by a two-fold rotation axis.

The relaxed structure of the core is pictured in Fig. 6. The structure initially appears to be little altered by the presence of the dislocation, although the core actually takes on a rather unusual structure. In order to understand this, it is necessary to consider the HCP oxygen sub-lattice and the nature of the discontinuity in the displacement field at the core of the screw dislocation. Looking along the incipient dislocation line in the perfect structure, one sees two triangles of oxygen ions "pointing" in opposite directions, where each triangle belongs to a different close packed layer and is offset from one another by $a / 2$ (Fig. 6b). These triangles form opposite faces of the M1
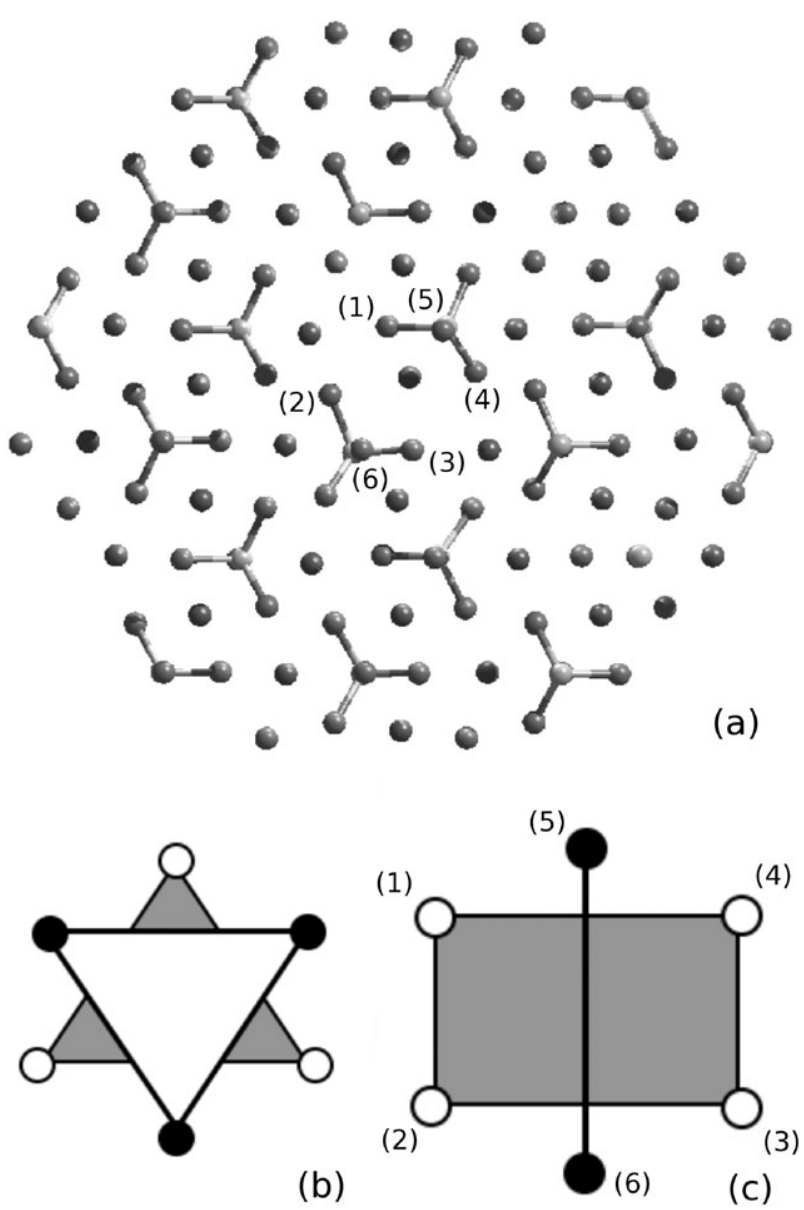

Fig. 6 View of the core of the $a[100]$ screw dislocation in forsterite (a) and a model for its structure ( $b$ and $c$ ). In part (a) magnesium (isolated ions), oxygen and silicon (forming tetrahedra) atoms are shown and only minor deformation can be seen from this view. Part (b) shows undeformed octahedra with atoms in the upper plane in black and the lower plane shown as white circles. Part (c) shows the arrangement after the introduction of the dislocation; atoms are numbered to allow comparison with part (a). Details of the three-dimensional structure of the core of this dislocation are available in xyz format as ESI. $f$

octahedra. On the introduction of the dislocation the oxygen atoms are displaced by an amount that depends on the angle around the dislocation line. The effect of this translation is shown in Fig. 6c. The resultant structure is rather unusual, in that it has a plane of four oxygen ions forming an almost square geometry, separated by pairs of atoms with the magnesium ions transferred into a six-coordinate, trigonal-prismatic environment.

Now turning to the core displacement field, Fig. S2, in the ESI, $\ddagger$ illustrates the components of this field perpendicular (S2a) and parallel (S2b) to the dislocation line. Along the dislocation line, there are some large displacements of oxygen ions, since they arrange themselves in the square conformation, with a correspondingly large displacement of the central $\mathrm{Mg}$ ion so that it can maintain six-fold co-ordination. It is worth noting that the magnitude of the displacements, as a fraction of the lattice vector, is much smaller than the displacements associated with the screw dislocation in $\mathrm{MgO}$. The displacements perpendicular to the dislocation line are predominantly inwards; presumably, this is to reduce the strain at the core associated with the introduction of the dislocation.

The methodology for modelling the $c[001]$ screw dislocation is identical to that described above for the $a[100]$ screw dislocation. Again, there are a limited number of sensible locations for the origin of the elastic displacement field avoiding the breaking of $\mathrm{Si}-\mathrm{O}$ bonds. The lowest energy starting configuration has the origin located along a string of M1 sites, 


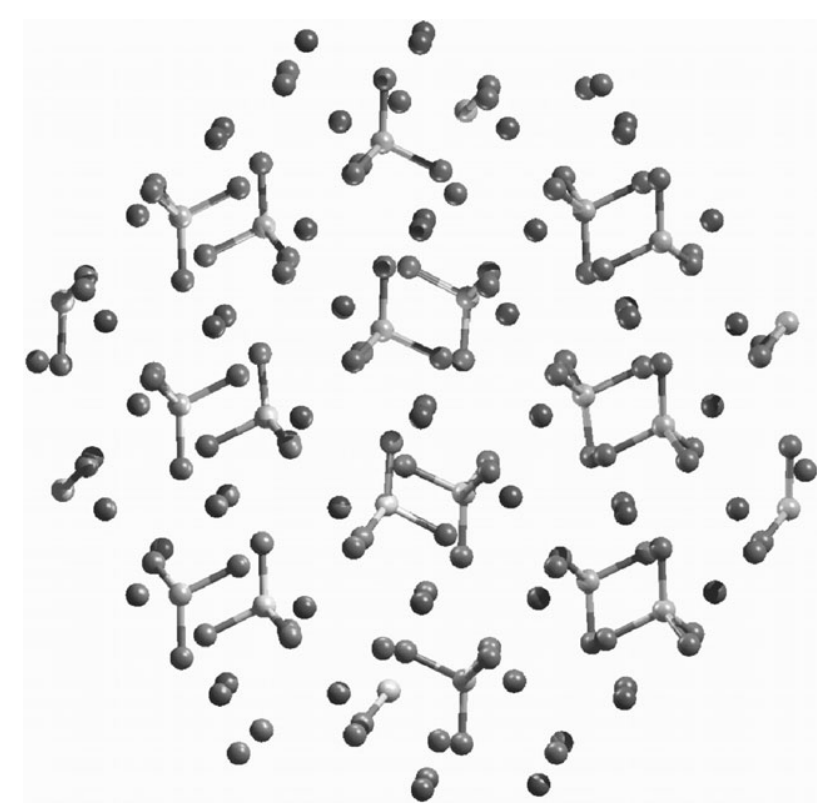

Fig. 7 View of the core of the $c[001]$ screw dislocation in forsterite The atom types correspond to those given in Fig. 7a and the structure is rather similar to the structure of bulk forsterite. Details of the threedimensional structure of the core of this dislocation are available in xyz format as ESI.

although the line does not pass directly through the octahedral faces.

Fig. 7 shows the structure of the core following relaxation. It is particularly difficult to visualize this structure in a twodimensional image, but the key change is that the string of inversion centres along the M1 sites becomes a two-fold rotational axis on the introduction of the dislocation. Examination of the structure reveals that all the magnesium ions are in octahedral coordination despite the presence of the dislocation core. The core displacement field is shown in Fig. S3, in the ESI, $\ddagger$ and the oxygen ions around the core move parallel to the dislocation line by up to $\sim 0.7 \AA(c / 8)$. The atoms also relax inwards, which corresponds to the expected strain relieving behaviour observed for the $a[100]$ screw dislocation in forsterite and $\mathrm{MgO}$. The $c / 8$ displacement parallel to the dislocation line results in a core structure that is very much like the undeformed crystal structure (see Fig. 8) by reversing the local effect of the screw dislocation close to the core.

Consideration of the geophysical significance of the qualitative differences between the predicted structure of the [100] and [001] screw dislocation cores is reserved for future work. However, we note that the structural differences could be the underlying reason for the apparent change in the olivine deformation mechanism at depths of about $250 \mathrm{~km}$ in the upper mantle ${ }^{20}$ and may lead to differences in the effect of the dislocations on the diffusion of ions through the crystal.

\section{Screw dislocations in zeolite $\mathbf{A}$}

In previous work, we described the possible structure of screw dislocations in zeolite $\mathrm{A}^{21}$ These screw dislocations enhance the growth rate under conditions of low supersaturation, resulting in the formation of growth spirals imaged by $\mathrm{AFM}^{22}$ and could open pathways for rapid diffusion into the crystal. Our calculations were based on a potential model making use of the Coulomb summation of Wolf et al. ${ }^{23}$ The simulation cell contained up to 250,000 atoms and charge neutrality was achieved by building the cell out of charge neutral $\left[\mathrm{Si}^{+4} \mathrm{O}_{4}^{-1}\right]$ units.

A comparison of the structural relaxation around the dislocation in the zeolite and in the two dense materials presented above is of particular interest. In the dense materials, ionic

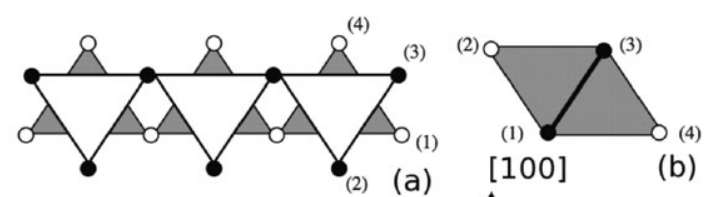

[010]

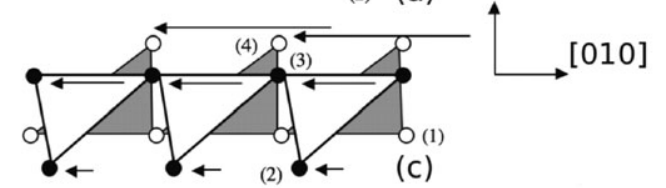

$[001]$

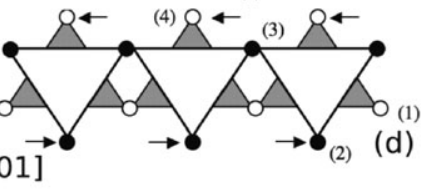

Fig. 8 Model for the structure of the core of a $c[001]$ screw dislocation in forsterite. Parts (a) and (b) show the structure of the oxygen sub lattice close to the incipient dislocation core in the direction looking along [100] and [001] respectively. Black atoms are close to the observer while white atoms are further away. Part (c) shows the relative motion of the atoms on the introduction of the dislocation, assuming atom (1) is taken as fixed, atom (2) moves by approximately $c / 4$, atom (3) moves by approximately $c / 2$ and atom (4) moves by approximately $3 c / 4$. Part $\mathrm{d}$ shows the atomistic relaxation around the core with atoms in strings 1 and 3 taken as fixed, this means that rather than displacing all the atoms by $c / 8$ (see Fig. 10a) two of the strings are displaced by $c / 4$. The atomistic relaxation along the $c$-axis results in the recreation of octahedral coordination for the magnesium ions.

relaxation perpendicular to the line of the screw dislocation is inwards as shown particularly clearly for $\mathrm{MgO}$ in Fig. S1a, but also for both forsterite dislocations (Figs. S2 and S3a). $\ddagger$ In the case of the zeolite the radial relaxation is a much smaller, both in absolute terms, and as a fraction of the Burgers vector, with the predominant radial relaxation being outwards (Fig. S4 in the ESI). $\ddagger$ Relaxation along the dislocation line is also smaller than in the dense materials $(\sim 0.8 \AA$, Fig. $12 \mathrm{~b}$, compared to 1.2 $\AA$ in $\mathrm{MgO}$, Fig. S1b, and $\sim 2 \AA$ in forsterite Figs. S2 and S3b), this is a very significant difference considered as a fraction of the Burgers vector. The small magnitude of these displacements is due to the origin of the dislocation being located along an empty channel in the zeolite structure, thus preventing the formation of a discontinuity in the elastic part of the displacement field. However, the bonds are extended by introducing the dislocation and part of the relaxation involves shortening these bonds by the rotation of quasi-rigid $\mathrm{SiO}_{4}$ units in the structure.

\section{Screw dislocations in paracetamol}

In our final example, we briefly consider the structure of screw dislocations in paracetamol ( $p$-hydroxyacetanilide, formula $\mathrm{OH}-\mathrm{C}_{6} \mathrm{H}_{4} \mathrm{NHCOCH}_{3}$ ), a widely used analgesic and antipyretic drug also known as acetaminophen. Three polymorphs are known, the stable form (form I) is monoclinic ${ }^{24}$ and is mixed with binders to produce commercially available tablets. Metastable form II is orthorhombic ${ }^{25}$ and is of current interest as an alternative to form I in tablet manufacture because tablets can be made by direct compression without a binding agent. ${ }^{26}$ Recently a second metastable form less stable than form II has been found. ${ }^{27-29}$

In particular, we consider the structure of the $c[001]$ screw dislocation in orthorhombic paracetamol-II (space group Pcab, Fig. 9). Selection of this, rather than one of the other polymorphs, including the more stable monoclinic form, is because of the relative ease of treating the elastic deformation in orthorhombic, compared to monoclinic crystals, and because of the reported difficulty in reproducing the morphology of paracetamol-II crystals. ${ }^{26,29}$ As a $c[001]$ screw dislocation is the perfect screw dislocation with the shortest Burgers vector, it 

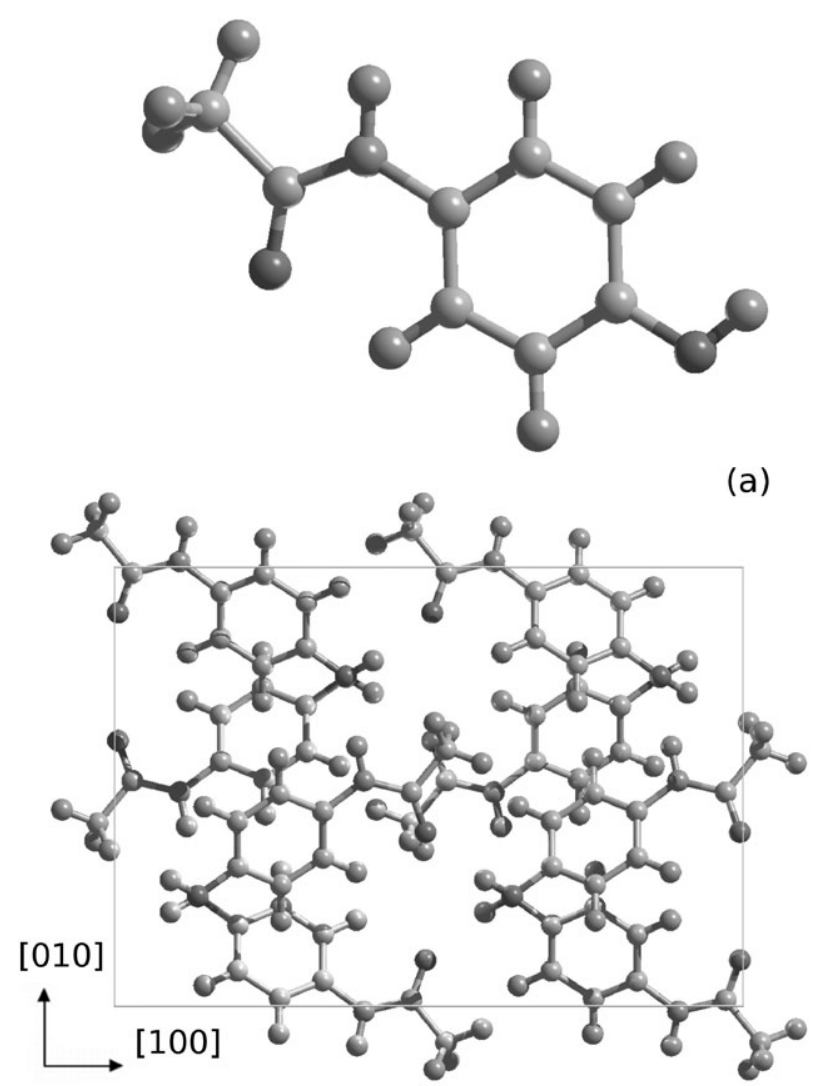

(b)

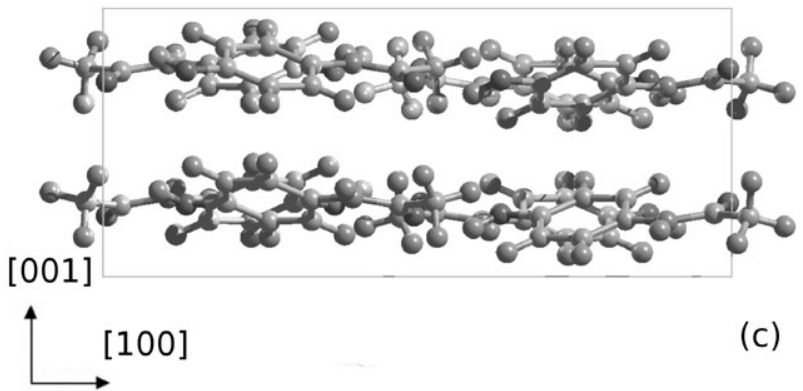

Fig. 9 Molecular and crystal structure of paracetamol-II. (a) Shows a single paracetamol molecule, (b) shows the crystal structure of paracetamol-II looking along the [001] axis at a hydrogen bonded layer and (c) views these layers edge on, looking along [010].

is predicted to have the lowest elastic energy and thus be the most common dislocation in the growing crystal. The presence of this dislocation would also help to explain the difficulty in reproducing morphology of paracetamol-II, because it would promote spiral growth on the (001) and (002) surfaces. This rationalises a major failing of morphology predictions based on the attachment energy, where the (002) surface is predicted to grow too slowly and is thus over expressed in morphologies predicted by the attachment energy model (see Fig. 8 of ref. 26). It is worth noting that diagnostic growth spirals on paracetamol-I have been observed using AFM, lending further support to this mechanism of crystal growth. ${ }^{30}$

Previous modelling, using connected net analysis, together with a Monte Carlo simulation has shown the critical importance of the spiral growth mechanism in paracetamol and demonstrated that the predicted morphology is force field dependent. ${ }^{31}$ As the spiral growth mechanism relies on the presence of dislocations, and dislocations can be detrimental to the compositional and structural uniformity of the drug, a detailed understanding of the core structure of dislocations is desirable and at the present time only computer modelling can hope to provide this.
Table 1 Comparison of the performance of our CVFF based forcefield with experimental and computational studies of the structure and properties of paracetamol-II

\begin{tabular}{lccc}
\hline & This work & $\begin{array}{l}\text { Previous } \\
\text { modelling } \\
\text { (ref. 29) }\end{array}$ & $\begin{array}{l}\text { X-ray } \\
\text { diffraction } \\
\text { (ref. 26) }\end{array}$ \\
\hline$a / \AA$ & 17.305 & 17.471 & $17.156(6)$ \\
$b / \AA$ & 11.547 & 11.877 & $11.831(4)$ \\
$c / \AA$ & 7.491 & 7.454 & $7.405(3)$ \\
& & & \\
$C_{11} / \mathrm{GPa}$ & 38.1 & 53.2 & \\
$C_{22} / \mathrm{GPa}$ & 29.5 & 26.3 & \\
$C_{33} / \mathrm{GPa}$ & 20.4 & 12.0 & \\
$C_{12} / \mathrm{GPa}$ & 4.1 & 21.2 & \\
$C_{13} / \mathrm{GPa}$ & 13.5 & 3.6 & \\
$C_{23} / \mathrm{GPa}$ & 18.5 & 8.3 & \\
$C_{44} / \mathrm{GPa}$ & 6.6 & 3.5 & \\
$C_{55} / \mathrm{GPa}$ & 0.1 & 0.7 & \\
$C_{66} / \mathrm{GPa}$ & 8.8 & 11.3 & \\
\hline
\end{tabular}

We describe paracetamol using a forcefield based on the CVFF scheme, ${ }^{32}$ as implemented in an early version of the InsightII code. ${ }^{33}$ The full forcefield contains cross terms that are omitted in this implementation, in order to reduce the number of terms considered, without significantly affecting the accuracy of the calculation. Our forcefield therefore contains harmonic two- and three-body bonded terms, selected torsional terms and Lennard-Jones non-bonded terms. The charges are assigned using the engine within version 3.1 of the Materials Studio software ${ }^{34}$ (which contains an updated form of the CVFF potential). In total, nine atom types were used to describe paracetamol: three hydrogen types, two oxygen, three carbon and one nitrogen, consistent with the CVFF forcefield and its implementation within the Accelrys codes.

We emphasise that for models of dislocations it is important to capture the correct lattice and elastic properties of the crystal. However, there have been no experimental determinations of the single crystal elastic constants of paracetamol-II. In Table 1, we therefore compare our calculated structure with an experimental determination of the room temperature cell parameters ${ }^{26}$ and elastic constants calculated using a significantly different computational methodology based on rigid molecules and a multipole expansion of the molecules' charge distribution. ${ }^{29}$ Key features of the elastic constant tensor are well reproduced, particularly the anisotropy associated with shearing the crystal perpendicular to the hydrogen bonded sheets (represented by the constants $\mathrm{C}_{44}$ and $\mathrm{C}_{55}$ ), which is crucial to describing the pitch of the screw dislocation.

The 1D simulation cell is built out of complete molecules of paracetamol in order to maintain charge neutrality. The cell has a radius of $50 \AA$ (any molecule with at least one atom outside this radius is completely removed), is periodic along the $c$ axis with a periodic repeat length of $7.491 \AA$, and contains 270 molecules of paracetamol. The cell is centred in the empty channel visible in Fig. 9b, located half way along the $a$ axis and quarter of the way along the $b$ axis. The screw dislocation was introduced utilising anisotropic elasticity, with elastic constants calculated from the potential model, and a Burgers vector of equal length to the $c$ axis. The cell was then relaxed to the energy minimum utilising a combination of the conjugate gradients and quasi-Newtonian optimisation algorithms, keeping atoms in the outer $20 \AA$ of the simulation cell fixed.

The major response of the crystal during relaxation is a change in the bond lengths and a movement of the paracetamol molecules perpendicular to the dislocation line. Upon introduction of the dislocation, assuming homogenous elastic properties, all bonds lengths (with the exception of any lying 


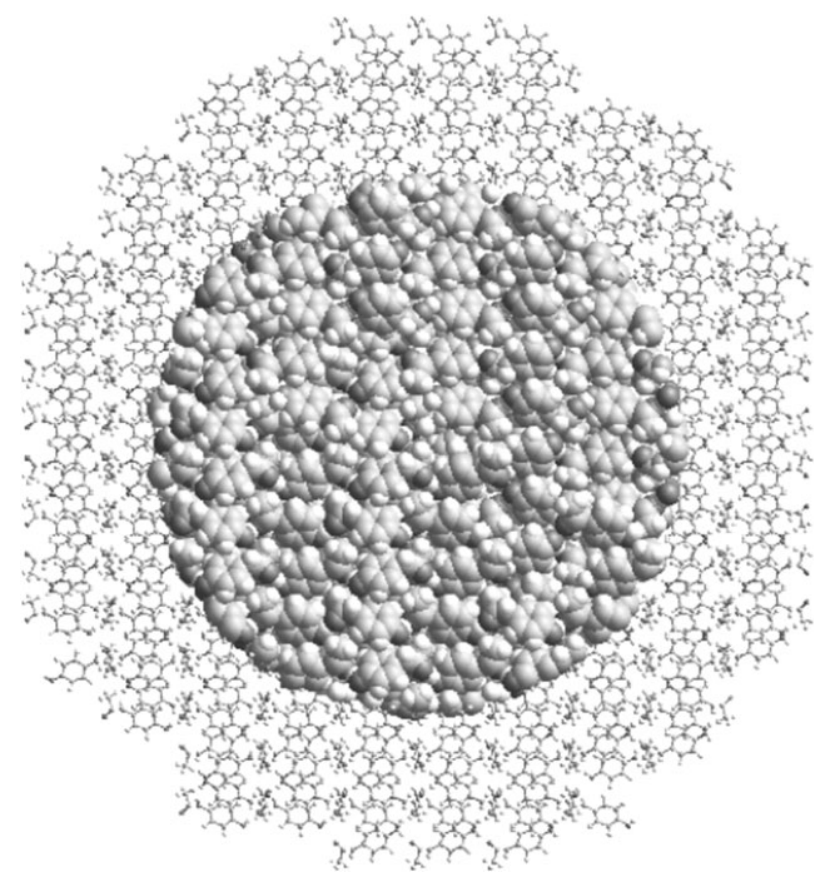

Fig. 10 View of the simulation cell used to study the core of the $c[001]$ screw dislocation in paracetamol-II. Atoms shown as large space filling spheres are relaxed to energy minimum during the simulation while those shown as stick models are held fixed at the location predicted by linear elastic theory. Details of the three-dimensional structure of the core of this dislocation are available in xyz format as ESI. $\neq$

parallel to the $c$ axis, of which there are none in paracetamolII) are changed. The magnitude of this change depends on the distance of the bond from the origin of the displacement field (larger distances decrease the change in length) and the relative orientation of the bond and the dislocation line. During subsequent energy minimisation, the bonds were observed to return to their original (perfect lattice) lengths as shown for the $\mathrm{C}-\mathrm{H}$ bonds in the benzene group in Fig. S5, in the ESI.t However, there is a small general increase in bond length in the dislocated crystal as compared to the perfect crystal. For instance, the $\mathrm{C}-\mathrm{H}$ bonds in the relaxed part of the dislocated crystal all lay within the range 1.065-1.076 $\AA$ and the dislocation free value is $1.078 \AA$.

The changing bond lengths mask the core displacement field associated with the relaxation-some atoms must move towards the dislocation line and others away from the dislocation line in order to change the bond lengths. In an attempt to deconvolute this contribution, we plot the displacement of the average position of each molecule in Fig. S6, in the ESI. $\ddagger$ While it is clear that the component of the core displacement field parallel to the dislocation line is small and rapidly diminishes, the average radial displacement is considerably larger and, for some of the molecules, is significant at the edge of the relaxed region.

Fig. 10 shows the simulation cell and details of the structure of the core can be found in the ESI. $\ddagger$ Away from the dislocation (or in a perfect crystal), a view perpendicular to the direction of the dislocation line would show the edges of two hydrogen bonded sheets of paracetamol molecules, shown in Fig. $9 \mathrm{~b}$. At the core of the dislocation, these sheets are twisted into a double helix around the dislocation line. In the perfect crystal, these sheets are held together by two types of hydrogen bond, $\mathrm{N}-\mathrm{H} \cdots \mathrm{O}$ with a calculated length of $1.713 \AA$ and $\mathrm{O}-\mathrm{H} \cdots \mathrm{O}$ with a length of $2.048 \AA$. Four of these hydrogen bonds form a closed loop around the void channel chosen as the origin of the dislocation. At the core, the hydrogen bond network is dramatically altered, the $\mathrm{O}-\mathrm{H} \cdots \mathrm{O}$ bond closest to the core is lengthened to $2.044 \AA$ (the second closest bond lengthens to $1.876 \AA$ ) while the two $\mathrm{N}-\mathrm{H} \cdots \mathrm{O}$ bonds are increased to 2.444 and $2.208 \AA$ for the closest and second closest bonds respectively. The original channel has a radius (defined as the distance between the $\mathrm{O}$ atoms in the amide group, across the cavity in each sheet) of $4.070 \AA$-the double helix brings these two atoms together into a plane perpendicular to $c$ and this distance is reduced to $3.407 \AA$.

The chemical effect of these structural changes is hard to predict without additional calculations to reveal the surface structure of the dislocated crystal. However, it is likely that the changes will be substantial, not least to the crystal growth assembly energetics at the dislocation. Breaking the $\mathrm{N}-\mathrm{H} \cdots \mathrm{O}$ hydrogen bond may induce additional reactivity to the crystal close to the dislocation core, possibly leading to the binding of solvent or impurity molecules to the growing surface where the dislocation emerges from the crystal. In future work, we will study the how binding of paracetamol and potential impurities is affected by the presence of these impurities.

\section{Conclusions}

We have extended an existing methodology for modelling screw dislocations and demonstrated its applicability for a wide range of crystals that show much structural and chemical complexity. It is interesting to contrast the reconstructions of the cores of the dislocations in the range of materials presented above. In $\mathrm{MgO}$, the reconstruction is a relatively simple smoothing of the discontinuity predicted by the elastic theory and results in a core structure that is similar to the structure of bulk $\mathrm{MgO}$. A similar type of reconstruction is observed for the $c$ [001] screw dislocation in forsterite, only in this case the magnitude of the reconstruction needed to smooth the discontinuity and return the core to a structure similar to that found in the dislocation free system is smaller (only $1 / 4$ of the Burgers vector rather than $1 / 2$ the Burgers vector in $\mathrm{MgO}$ ). In contrast the structure of the core of the $a[100]$ screw dislocation is significantly different to dislocation free forsterite, possibly because much more significant reconstructions would be needed to return to a bulk like configuration and these would be associated with considerable elastic penalties. Turning to the lower density materials, in zeolite A there is very little reconstruction at the core of the dislocation. This is due to the open channel structure of the zeolite removing the necessity of a discontinuity across the core of the dislocation, with the result that the elastic description of the atomic displacements is a good approximation of the atomistic structure. In the case of paracetamol the reconstruction is more difficult to describe as it is masked by a reduction in strain of all the molecules in the model. However, the displacements of the molecules close to the core do not appear to be particularly large. This may be due to the location of the origin of the displacement field along a significant void channel in the structure.

It is also worth considering the relative energetic cost of introducing the dislocations into these materials. For macroscopic crystals, almost all of this energy is associated with the elastic deformation of the crystal away from the core and this is proportional to the square of the length of the Burgers vector and (in cubic and orthorhombic crystals) the product two elastic constants. Thus, we expect soft materials, such as paracetamol, to have significantly lower total dislocation formation energies than stiff materials such as $\mathrm{MgO}$ and forsterite. Consequently, we expect the incidence of screw dislocations to be relatively high in many weakly bound, soft materials, like pharmaceuticals. However, there have been relatively few reported AFM or TEM investigations on this class of materials, so this speculation cannot be confirmed.

Further advances are anticipated, in particular we have already demonstrated the application of the general methodology outlined above to studies of edge dislocations in forsterite $^{35}$ and studies of dislocations with a mixed character are, in principle, possible. We anticipate the implementation of one of 
the schemes that allow the periodic update of the co-ordinates of the outer sheath of atoms (flexible boundary conditions), which will solve some of the problems associated with the fixed boundary method noted above. Such a scheme would also be useful in describing moving dislocations.

We expect that the approach described in these papers will be of utility in a number of fields where the properties of dislocations in a wide variety of materials are important. These include their clear action as agents of crystal growth and plastic deformation but also in other materials, for example, zeolite A where models of dislocations have yielded hitherto unforeseen properties. In order to perform studies of a wide range of materials we intend to integrate the modelling methodology into the open source GDis visualisation software. ${ }^{36}$

\section{Acknowledgements}

We thank EPSRC for funding access to local computer resources (GR/S06233/01 and GR/S84446/01), access to national supercomputing facilities via the Materials Chemistry Consortium (GR/S13422/01) and a studentship for A.M.W. K.W. thanks the Royal Society for support under their University Research Fellowship Scheme and J.D.G. gratefully acknowledges the support of the Government of Western Australia through a Premier's Research Fellowship.

\section{References}

1 A. M. Walker, J. D. Gale, B. Slater and K. Wright, Phys. Chem. Chem. Phys., 2005, 7, DOI: 10.1039/b505612h.

2 J. D. Gale and A. L. Rohl, Mol. Simul., 2003, 29, 291-341.

3 G. V. Lewis and C. R. A. Catlow, J. Phys. C, 1985, 18, 1149-1161.

4 (a) C. R. A. Catlow, D. A. Faux and M. J. Norgett, J.Phys. C, 1976, 9, 419-429; (b) M. Matsui, S. C. Parker and M. Leslie, Am Miner., 2000, 85, 316-400; (c) M. Matsui, J. Chem. Phys., 1998, 108, 3304-3309.

5 H. J. Frost and M. F. Ashby, Deformation Mechanism Maps. The Plasticity and Creep of Metals and Ceramics, Pergamon Press, Oxford, 1982.

6 G. W. Watson, P. M. Oliver and S. C. Parker, Surf. Sci. Lett., 2001, 474, L185-L190.

7 G. W. Watson, E. T. Kelsey and S. C. Parker, Philos. Mag. A, 1999, 79, 527-536.

8 G. W. Watson, E. T. Kelsey, N. H. de Leeuw, D. J. Harris and S. C. Parker, J. Chem. Soc., Faraday Trans., 1996, 92, 433-438.

9 M. P. Puls and M. J. Norgett, J. Appl. Phys., 1976, 47, 466- 477.

10 R. G. Hoagland, J. P. Hirth and P. C. Gehlen, Philos. Mag., 1976 34, 413-439.

11 V. R. Saunders, C. Freyria-Fava, R. Dovesi and C. Roetti, Comput. Phys. Commun., 1994, 84, 156-172.

12 J. W. Steeds, Introduction to Anisotropic Elasticity Theory of Dislocations, Oxford University Press, Oxford, 1973.

13 (a) M. Wilson, Chem. Phys. Lett., 2002, 366, 504-509; (b) E. Philp, J. Sloan, A. I. Kirkland, R. R. Meyer, S. Friedrichs, J. L. Hutchinson and M. L. H. Green, Nat. Mater., 2003, 2, 788-791.

14 O. Jaoul, J. Geophys. Res., 1990, 95, 17631-17642.

15 J.-P. Poirier, J. Geophys. Res., 1975, 80, 4059-4061.
16 (a) P. Cordier, in Plastic Deformation and Deformation Microstructure in Earth Materials, ed. S.-I. Karato and H.-R. Wenk, Mineralogical Society of America, Washington, 2002, pp. 137179; (b) W. B. Durham, C. Goetze and B. Blake, J. Geophys. Res., 1977, 82, 5755-5770; (c) Y. Gueguen and M. Darot, Philos. Mag. $A, 1982,45,419-442 ;(d)$ S. Mei and D. L. Kohlstedt, J. Geophys. Res., 2000, 105, 21471-21481; (e) C. L. Johnson, M. J. Hÿtch and P. R. Buseck, Am. Miner., 2004, 89, 1374-1379.

17 (a) M. J. Sanders, M. Leslie and C. R. A. Catlow, J. Chem. Soc., Chem. Commun., 1984; (b) C. R. A. Catlow, Proc. R. Soc. London, Ser. A, 1977, 353, 533-561.

18 (a) G. D. Price, S. C. Parker and M. Leslie, Phys. Chem. Miner. 1987, 15, 181-190; (b) G. D. Price, S. C. Parker and M. Leslie, Mineral. Mag., 1987, 51, 157-170; (c) S. C. Parker and G. D. Price, Adv. Solid-State Chem., 1989, 1, 295-327; (d) O. Jaoul, Y. BertranAlvarez, R. C. Liebermann and G. D. Price, Phys. Earth Planet. Inter., 1995, 89, 199-218; (e) N. H. de Leeuw, S. C. Parker, C. R. A. Catlow and G. D. Price, Phys. Chem. Miner, 2000, 27, 332 341; (f) N. H. de Leeuw, S. C. Parker, C. R. A. Catlow and G. D. Price, Am. Miner., 2000, 85, 1143-1154; $(g)$ K. Wright and C. R. A. Catlow, Phys. Chem. Miner., 1994, 20, 515-518; (h) A. M. Walker, K. Wright and B. Slater, Phys. Chem. Miner., 2003, 30, 536-545; (i) J. A. Purton, N. L. Allan and J. D. Blundy, Geochim. Cosmochim. Acta, 1997, 61, 3927-3936; (j) G. W. Watson, P. M. Oliver and S. C. Parker, Phys. Chem. Miner., 1997, 25, 70-78; $(k)$ J. A. Purton, N. L. Allan, J. D. Blundy and E. A. Wasserman, Geochim. Cosmochim. Acta, 1996, 60, 4977-4987.

19 J. S. Braithwaite, P. V. Sushko, K. Wright and C. R. A. Catlow, J. Chem. Phys., 2002, 116, 2628-2635.

20 D. Mainprice, A. Tommasi, H. Couvy, P. Cordier and D. Frost, Nature, 2005, 433, 731-733.

21 A. M. Walker, B. Slater, J. D. Gale and K. Wright, Nat. Mater., 2004, 3, 715-720.

22 S. Dumrul, S. Bazzana, J. Warzywoda, R. R. Biederman and A Sacco, Microporous Mesoporous Mat., 2002, 54, 79-88.

23 D. Wolf, P. Keblinski, S. R. Phillpot and J. Eggebrecht, J. Chem. Phys., 1999, 110, 8254-8282.

24 M. Haisa, S. Kashino and H. Maeda, Acta Crystallogr., Sect. B, 1976, B32, 1283-1285.

25 M. Haisa, S. Kashino and H. Maeda, Acta Crystallogr., Sect. B, 1974, B30, 2510-2513.

26 G. Nichols and C. S. Frampton, J. Pharm. Sci., 1998, 87, 684-693.

27 A. Burger, Acta Pharm. Technol., 1982, 28, 1-20.

28 M. L. Peterson, S. L. Morissette, C. McNulty, A. Goldsweig, P. Shaw, M. LeQuesne, J. Monagle, N. Encina, J. Marchionna, A. Johnson, J. Gonzalez-Zugasti, A. V. Lemmo, S. J. Ellis, M. J. Cima and Ö. Almarsson, J. Am. Chem. Soc., 2002, 124, 10958 10959.

29 T. Beyer, G. M. Day and S. L. Price, J. Am. Chem. Soc., 2001, 123, 5086-5094.

30 C. M. Chew, R. I. Ristic, R. D. Dennehy and J. J. De Yoreo, Cryst. Growth Des., 2004, 4, 1045-1052.

31 H. M. Cuppen, G. M. Day, P. Verwer and H. Meekes, Cryst. Growth Des., 2004, 4, 1341-1349.

32 A. T. Hagler, P. Lifson and P. Dauber, J. Am. Chem. Soc., 1979, 101, 5122-5130.

33 InsightII version $300^{\circledR}$, Accelrys ${ }^{\circledR}$ (formerly Biosym Technologies), USA.

34 Materials Studio ${ }^{\circledR}$ Modeling 3.1 software suite, Accelrys ${ }^{\circledR}$, USA

35 A. M. Walker, PhD Thesis, University of London, 2004.

36 S. Fleming and A. Rohl, Z. Krist., 2005, 220, 580-584. 